\title{
ON ASYMPTOTICALLY MINIMAX NONPARAMETRIC DETECTION \\ OF SIGNAL IN GAUSSIAN WHITE NOISE
}

\author{
MIKHAIL ERMAKOV
}

erm2512@gmail.com

Institute of Problems of Mechanical Engineering, RAS and

St. Petersburg State University, St. Petersburg, RUSSIA

Mechanical Engineering Problems Institute

Russian Academy of Sciences

Bolshoy pr.,V.O., 61

St.Petersburg

Russia

St.Petersburg State University

University pr., 28, Petrodvoretz

198504 St.Petersburg

Russia

St.Petersburg department of

Steklov mathematical institute

Fontanka 27, St.Petersburg 191023

\begin{abstract}
For the problem of nonparametric detection of signal in Gaussian white noise we point out strong aasymptotically minimax tests. The sets of alternatives are a ball in Besov space $B_{2 \infty}^{r}$ with "small" balls in $L_{2}$ removed.
\end{abstract}

keyword 1 (class=AMS). [Primary ]62G10,62G20,62M02

keyword 2. signal detection, asymptotic minimaxity, asymptotic efficiency, Gaussian white noise

\section{Introduction. Main Result.}

Let we observe a random process $Y_{\epsilon}(t), t \in(0,1), \epsilon>0$, defined by stochastic differential equation

$$
d Y_{\epsilon}(t)=\theta(t) d t+\epsilon d w(t)
$$

with Gaussian white noise $w(t)$. The signal $\theta \in L_{2}(0,1)$ is unknown.

Our goal is to test the hypothesis

$$
H_{0}: \theta(t)=0, \quad t \in(0,1)
$$

versus the alternative

$$
H_{\epsilon}: \quad \int_{0}^{1} \theta^{2}(t) d t=\|\theta\|^{2}>\rho_{\epsilon}>0
$$

if a priori information is provided that

$$
\theta \in B_{2 \infty}^{r}\left(P_{0}\right)=\left\{\theta: \theta(t)=\sum_{j=1}^{\infty} \theta_{j} \phi_{j}(t), k^{-2 r} \sum_{j=k}^{\infty} \theta_{j}^{2} \leq P_{0}, t \in(0,1), 1 \leq k<\infty\right\}
$$


with $P_{0}>0$. Here $\phi_{j}, 1 \leq j<\infty$, is orthonormal system of functions. For wide class of orthonormal systems of functions $\phi_{j}, 1 \leq j<\infty$ the space

$$
\left\{\theta: \theta(t)=\sum_{j=1}^{\infty} \theta_{j} \phi_{j}(t), k^{-2 r} \sum_{j=k}^{\infty} \theta_{j}^{2}<\infty, t \in(0,1), 1 \leq k<\infty\right\}
$$

is Besov space $B_{2 \infty}^{r}$ (see [8])

Denote $Q_{\epsilon}=\left\{\theta:\|\theta\|^{2} \geq \rho_{\epsilon}, \theta \in B_{2 \infty}^{r}\left(P_{0}\right)\right\}$.

For any test $K \epsilon$ denote $\alpha\left(K_{\epsilon}\right)$ its type I error probability and denote $\beta_{\theta}\left(K_{\epsilon}\right)$ its type II error probability for the alternative $\theta \in Q_{\epsilon}$.

We put

$$
\beta_{\epsilon}\left(K_{\epsilon}\right)=\sup _{\theta \in Q_{\epsilon}} \beta_{\theta}\left(K_{\epsilon}\right) .
$$

We say that family of tests $L_{\epsilon}$ is asymptotically minimax if, for any family of tests $K_{\epsilon}, \alpha\left(K_{\epsilon}\right) \leq \alpha\left(L_{\epsilon}\right)$, there holds

$$
\limsup _{\epsilon \rightarrow 0} \beta_{\epsilon}\left(K_{\epsilon}\right)-\beta_{\epsilon}\left(L_{\epsilon}\right) \geq 0 .
$$

Paper goal is to establish asymptotically minimax families of tests $L_{\epsilon}$ for the sets of alternatives $Q_{\epsilon}$. If the sets of alternatives are ellipsoids with "small balls" removed, asymptotically minimax families of tests have been found in [2]. For nonparametric hypothesis testing this result can be considered as a version of Pinsker Theorem 6. 7, 5] on asymptotically minimax nonparametric estimation. Note that hypothesis testing with nonparametric sets of alternatives belonging some ball in functional space is intensively studied (see 4, 1] and references therein).

The proof, in main features, repeats the reasoning in [2]. The main difference in the proof is the solution of another extremal problem minimizing type II error probabilities caused another definition of sets of alternatives. Other differences have technical character and are also caused the differences of definitions of sets of alternatives.

Define $k=k_{\epsilon}$ and $\kappa^{2}=\kappa_{\epsilon}^{2}$ as a solution of two equations

$$
2 r k_{\epsilon}^{2 r+1} \kappa_{\epsilon}^{2}=P_{0}
$$

and

$$
k_{\epsilon} \kappa_{\epsilon}^{2}+k_{\epsilon}^{-2 r} P_{0}=\rho_{\epsilon} .
$$

Denote $\kappa_{j}^{2}=\kappa_{\epsilon}^{2}$, for $1 \leq j \leq k_{\epsilon}$ and $\kappa_{j}^{2} P_{0}(2 r)^{-1} j^{-2 r-1}$, for $j>k_{\epsilon}$.

Define test statistics

$$
T_{\epsilon}^{a}\left(Y_{\epsilon}\right)=\epsilon^{-4} \sum_{j=1}^{\infty} \kappa_{j}^{2} y_{j}^{2}
$$

$$
A_{\epsilon}=\epsilon^{-4} \sum_{j=1}^{\infty} \kappa_{j}^{4}
$$

For type I error probabilities $\alpha, 0<\alpha<1$, define critical regions

$$
S_{\epsilon}^{a}==\left\{y:\left(T_{\epsilon}^{a}(y)-\epsilon^{-2} \rho_{\epsilon}\right)\left(2 A_{\epsilon}\right)^{-1 / 2}>x_{\alpha}\right\}
$$

with $x_{\alpha}$ defined by equation

$$
\alpha=1-\Phi\left(x_{\alpha}\right)=(2 \pi)^{-1 / 2} \int_{x_{\alpha}}^{\infty} \exp \left\{-t^{2} / 2\right\} d t .
$$

Theorem 1.1. Let

$$
0<\lim \inf _{\epsilon \rightarrow 0} A_{\epsilon} \leq<\lim \sup _{\epsilon \rightarrow 0} A_{\epsilon}<\infty .
$$


Then the tests $L_{\epsilon}^{a}$ with critical regions $S_{\epsilon}^{a}$ are asymptotically minimax with $\alpha\left(L_{\epsilon}^{a}\right)=$ $\alpha(1+o(1))$ and

$$
\beta_{\epsilon}\left(L_{\epsilon}^{a}\right)=\Phi\left(x_{\alpha}-\left(A_{\epsilon} / 2\right)^{1 / 2}\right)(1+o(1))
$$

as $\epsilon \rightarrow 0$.

Example. Let $\rho_{\epsilon}=R \epsilon^{\frac{8 \beta}{4 \beta+1}}$. Then

$$
A_{\epsilon}=\left(\frac{P_{0}}{2 r}\right)^{1 / 2 r} \frac{4 r+2}{4 r+1}\left(\frac{R}{2 r+1}\right)^{\frac{4 r-1}{2 r}} .
$$

In what follows, we shall denote letter $C$ and $C$ with indices different generic constants.

\section{Proof of Theorem 1.1}

Fix $\delta, 0<\delta<1$. Denote $\kappa_{j}^{2}(\delta)=0$ for $j>\delta^{-1} k_{\epsilon}$. Define $\kappa_{j}^{2}(\delta), 1 \leq j<k_{\epsilon}=$ $\delta^{-1} k_{\epsilon}$, the equations (1.2) and (1.3) with $P_{0}$ and $\rho_{\epsilon}$ replaced with $P_{0}(1-\delta)$ and $\rho_{\epsilon}(1+\delta)$ respectively. Similarly to [2], we find Bayes test for a priori distribution $\theta_{j}=\eta_{j}=\eta_{j}(\delta), 1 \leq j<\infty$, with Gaussian independent random variables $\eta_{j}, E \eta_{j}=$ $0, E \eta_{j}^{2}=\kappa_{j}^{2}(\delta)$ and show that this test is asymptotically minimax for some $\delta=\delta_{\epsilon} \rightarrow$ 0 as $\epsilon \rightarrow 0$.

Lemma 2.1. For any $\delta, 0<\delta<1$, there holds

$$
P\left(\eta(\delta)=\left\{\eta_{j}(\delta)\right\}_{j=1}^{\infty} \in Q_{\epsilon}\right)=1+o(1)
$$

as $\epsilon \rightarrow 0$.

Denote

$$
A_{\epsilon, \delta}=\epsilon^{-4} \sum_{j=1}^{\infty} \kappa_{j}^{4}(\delta) .
$$

By straightforward calculations, we get

$$
\lim _{\delta \rightarrow 0} \lim _{\epsilon \rightarrow 0} A_{\epsilon} A_{\epsilon}^{-1}(\delta)=1 .
$$

Denote $\gamma_{j}^{2}(\delta)=\kappa_{j}^{2}(\delta)\left(\epsilon^{2}+\kappa_{j}^{2}(\delta)\right)^{-1}$.

By Neymann-Pearson Lemma the Bayes critical region is defined the inequality

$$
\begin{aligned}
& C_{1}<\prod_{j=1}^{k_{\epsilon \delta}}(2 \pi)^{-1 / 2} \kappa_{j}^{-1}(\delta) \int \exp \left\{-\sum_{j=1}^{k_{\epsilon \delta}}\left(2 \gamma_{j}^{2}(\delta)\right)^{-1}\left(u_{j}-\gamma_{j}^{2}(\delta) y_{j}\right)^{2}\right\} \exp \left\{-T_{\epsilon \delta}(y)\right\} \\
& =\exp \left\{-T_{\epsilon \delta}(y)\right\}(1+o(1))
\end{aligned}
$$

where

Define critical region

$$
T_{\epsilon \delta}(y)=\epsilon^{-2} \sum_{j=1}^{\infty} \gamma_{j}^{2}(\delta) y_{j}^{2}
$$

$$
S_{\epsilon \delta}=\left\{y: R_{\epsilon \delta}(y)=\left(T_{\epsilon \delta}(y)-C_{\epsilon \delta}\right)\left(2 A_{\epsilon}(\delta)\right)^{-1 / 2}>x_{\alpha}\right\}
$$

with

$$
C_{\epsilon \delta}=E_{0} T_{\epsilon \delta}(y)=\epsilon^{-2} \sum_{j=1}^{\infty} \gamma_{j}^{2}(\delta) .
$$

Denote $L_{\epsilon \delta}$ the tests with critical regions $S_{\epsilon \delta}$.

Denote $\gamma_{j}^{2}=\kappa_{j}^{2}\left(\epsilon^{2}+\kappa_{j}^{2}\right)^{-1}, 1 \leq j<\infty$ Define test statistics $T_{\epsilon}, R_{\epsilon}$, critical regions $S_{\epsilon}$ and constants $C_{\epsilon}$ by the same way as test statistics $T_{\epsilon \delta}, R_{\epsilon \delta}$, critical regions $S_{\epsilon \delta}$ and constants $C_{\epsilon, \delta}$ respectively with $\gamma_{j}^{2}(\delta)$ replaced with $\gamma_{j}^{2}$ respectively. Denote $L_{\epsilon}$ the test having critical region $S_{\epsilon}$. 
Lemma 2.2. Let $H_{0}$ hold. Then the distributions of tests statistics $R_{\epsilon}^{a}(y)$ and $R_{\epsilon}(y)$ converge to the standard normal distribution.

For any family $\theta_{\epsilon}=\left\{\theta_{j \epsilon}\right\} \in Q_{\epsilon}$ there holds

$$
P_{\theta_{\epsilon}}\left(\left(T_{\epsilon}^{a}(y)-\epsilon^{-2} \rho_{\epsilon}-\epsilon^{-4} \sum_{j=1}^{\infty} \kappa_{j}^{2} \theta_{j \epsilon}^{2}\right)\left(2 A_{\epsilon}\right)^{-1 / 2}<x_{\alpha}\right)=\Phi\left(x_{\alpha}\right)(1+o(1)) .
$$

and

$$
P_{\theta_{\epsilon}}\left(\left(T_{\epsilon}(y)-C_{\epsilon}-\epsilon^{-4} \sum_{j=1}^{\infty} \kappa_{j}^{2} \theta_{j \epsilon}^{2}\right)\left(2 A_{\epsilon}\right)^{-1 / 2}<x_{\alpha}\right)=\Phi\left(x_{\alpha}\right)(1+o(1)) .
$$

as $\epsilon \rightarrow 0$.

Hence we get the following Lemma.

Lemma 2.3. There holds

$$
\beta_{\epsilon}\left(L_{\epsilon}\right)=\beta_{\epsilon}\left(L_{\epsilon}^{a}\right)(1+o(1))
$$

as $\epsilon \rightarrow 0$.

Lemma 2.4. Let $H_{0}$ hold. Then the distribution of tests statistics $\left(T_{\epsilon}(y)-\right.$ $\left.C_{\epsilon \delta}\right)\left(2 A_{\epsilon}\right)^{-1 / 2}$ converge to the standard normal distribution.

There holds

$$
P_{\eta(\delta)}\left(\left(T_{\epsilon \delta}(y)-C_{\epsilon \delta}-A_{\epsilon \delta}\right)\left(2 A_{\epsilon \delta}\right)^{-1 / 2}<x_{\alpha}\right)=\Phi\left(x_{\alpha}\right)(1+o(1)) .
$$

as $\epsilon \rightarrow 0$.

Lemma 2.5. There holds

$$
\lim _{\delta \rightarrow 0} \lim _{\epsilon \rightarrow 0} E_{\eta(\delta)} \beta_{\eta(\delta)}\left(L_{\epsilon \delta}\right)=\lim _{\epsilon \rightarrow 0} E_{\eta_{0}} \beta_{\eta_{0}}\left(L_{\epsilon}\right)
$$

where $\eta_{0}=\left\{\eta_{0 j}\right\}_{j=1}^{\infty}$ and $\eta_{0 j}$ are i.i.d. Gaussian random variables, $E \eta_{0 j}=0, \eta_{0 j}^{2}=$ $\kappa_{j}^{2}, 1 \leq j<\infty$.

Define Bayes a priori distribution $P_{y}$ as a conditional distribution of $\eta$ given $\eta \in Q_{\epsilon}$. Denote $K_{\epsilon}=K_{\epsilon \delta}$ Bayes test with Bayes a priori distribution $P_{y}$. Denote $V_{\epsilon}$ critical region of $K_{\epsilon \delta}$.

For any sets $A$ and $B$ denote $A \triangle B=(A \backslash B) \cup(B \backslash A)$.

Lemma 2.6. There holds

$$
\lim _{\delta \rightarrow 0} \lim _{\epsilon \rightarrow 0} \int_{Q_{\epsilon}} P_{\theta}\left(S_{\epsilon \delta} \triangle V_{\epsilon \delta}\right) d P_{y}=0
$$

and

$$
\lim _{\delta \rightarrow 0} \lim _{\epsilon \rightarrow 0} P_{0}\left(S_{\epsilon \delta} \triangle V_{\epsilon \delta}\right)=0 .
$$

In the proof of Lemma 2.6 we show that the integrals in the right hand-side of (2.3) with integration domain $Q_{\epsilon}$ converge to one in probability as $\epsilon \rightarrow 0$. This statement is proved both for hypothesis and Bayes alternative (see [2]).

Lemmas 2.1 2.6 implies that, if $\alpha\left(K_{\epsilon}\right)=\alpha\left(L_{\epsilon}\right)$, then

$$
\int_{Q_{\epsilon}} \beta_{\theta}\left(K_{\epsilon}\right) d P_{y}=\int_{Q_{\epsilon}} \beta_{\theta}\left(L_{\epsilon}\right) d P_{y}(1+o(1))=\int \beta_{\eta_{0}}\left(L_{\epsilon}\right) d P_{\eta_{0}}(1+o(1)) .
$$

Lemma 2.7. There holds

$$
E_{\eta_{0}} \beta_{\eta_{0}}\left(L_{\epsilon}\right)=\beta_{\epsilon}\left(L_{\epsilon}\right)(1+o(1)) .
$$

Lemmas 2.2, 2.5, (2.2), (2.11) and Lemma 2.7 imply Theorem 1.1. 
ON ASYMPTOTICALLY MINIMAX NONPARAMETRIC DETECTIONOF SIGNAL IN GAUSSIAN WHITE NOISE

\section{Proof of Lemmas}

Proofs of Lemmas 2.2 2.3 and 2.5 are akin to the proofs of similar statements in [2] and are omitted.

Proof of Lemma 2.1. By straightforward calculations, we get

$$
\sum_{j=1}^{\infty} E \eta_{j}^{2}(\delta) \geq \rho_{\epsilon}(1+\delta / 2)
$$

and

$$
\operatorname{Var}\left(\sum_{j=1}^{\infty} \eta_{j}^{2}(\delta)\right)<C A_{\epsilon} \epsilon^{-4} \asymp \rho_{\epsilon}^{2} k_{\epsilon}^{-1} .
$$

Hence, by Chebyshev inequality, we get

$$
P\left(\sum_{j=1}^{\infty} \eta_{j}^{2}(\delta)>\rho_{\epsilon}\right)=1+o(1)
$$

as $\epsilon \rightarrow 0$. It remains to estimate

$$
P_{\mu}\left(\eta \notin B_{2 \infty}^{r}\right)=P\left(\max _{l_{1} \leq i \leq l_{2}} i^{2 r} \sum_{j=i}^{l_{2}} \eta_{j}^{2}-P_{0}\left(1-\delta_{1} / 2\right)>P_{0} \delta_{1} / 2\right) \leq \sum_{i=l_{1}}^{l_{2}} J_{i}
$$

with

$$
J_{i}=P\left(i^{2 r} \sum_{j=i}^{l_{2}} \eta_{j}^{2}-P_{0}\left(1-\delta_{1} / 2\right)>P_{0} \delta_{1} / 2\right)
$$

To estimate $J_{i}$ we implement the following Proposition [3]

Proposition 3.1. Let $\xi=\left\{\xi_{i}\right\}_{i=1}^{l}$ be Gaussian random vector with i.i.d.r.v.'s $\xi_{i}$, $E\left[\xi_{i}\right]=0, E\left[\xi_{i}^{2}\right]=1$. Let $A \in R^{l} \times R^{l}$ and $\Sigma=A^{T} A$. Then

$$
P\left(\|A \xi\|^{2}>\operatorname{tr}(\Sigma)+2 \sqrt{\operatorname{tr}\left(\Sigma^{2}\right) t}+2\|\Sigma\| t\right) \leq \exp \{-t\} .
$$

We put $\Sigma_{i}=\left\{\sigma_{l j}\right\}_{l, j=i}^{k_{\epsilon \delta}}$ with $\sigma_{j j}=j^{-2 r-1} i^{2 r} \frac{P_{0}-\delta}{2 r}$ and $\sigma_{l j}=0$ if $l \neq j$.

Let $i \leq k_{\epsilon}$. Then

$$
\operatorname{tr} \Sigma_{i}^{2}=i^{4 r} \sum_{j=i}^{\infty} \kappa_{j}^{4}(\delta)<i^{4 r}\left(\left(k_{\epsilon}-i\right) \kappa^{4}(\delta)+k_{\epsilon}^{-4 r-1} P_{0}\right)<C k_{\epsilon}^{-1} .
$$

and

Therefore

$$
\left\|\Sigma_{i}\right\| \leq i^{2 r} \kappa^{2}<C k_{\epsilon}^{-1}
$$

$$
2 \sqrt{\operatorname{tr}\left(\Sigma_{i}^{2}\right) t}+2\left\|\Sigma_{i}\right\| t \leq C\left(\sqrt{k_{\epsilon}^{-1} t}+k_{\epsilon}^{-1} t\right)
$$

Hence, putting $t=k_{\epsilon}^{1 / 2}$, by Proposition 3.1, we get

$$
\sum_{i=1}^{k_{\epsilon}} J_{i} \leq C k_{\epsilon} \exp \left\{-C k_{\epsilon}^{1 / 2}\right\} \text {. }
$$

Let $i \geq k_{\epsilon}$. Then

$$
\operatorname{tr} \Sigma_{i}^{2}<C i^{-1}, \quad \text { and } \quad\left\|\Sigma_{i}\right\| \leq C i^{-1}
$$

Hence, putting $t=i^{1 / 2}$, by Proposition 3.1. we get

$$
\sum_{i=k_{\epsilon}+1}^{k_{\epsilon \delta}} J_{i} \leq \sum_{i=k_{\epsilon}+1}^{k_{\epsilon \delta}} \exp \left\{-C i^{1 / 2}\right\}<\exp \left\{-C_{1} k_{\epsilon}^{1 / 2}\right\} .
$$

Now (3.4), (3.9), (3.11) together implies Lemma 2.1. 
Proof of Lemma 2.6. By reasoning of the proof of Lemma 4 in [2, Lemma 2.6 will be proved if we show that

$$
P\left(\sum_{j=1}^{\infty}\left(\eta_{j}(\delta)+y_{j} \gamma_{j}(\delta) \epsilon^{-1}\right)^{2}>\rho_{\epsilon}\right)=1+o(1)
$$

and

$$
P\left(\sup _{i} i^{2 r} \sum_{j=i}^{\infty}\left(\eta_{j}(\delta)+y_{j} \gamma_{j}(\delta) \epsilon^{-1}\right)^{2}>\rho_{\epsilon}\right)=1+o(1)
$$

where $y_{j}, 1 \leq j<\infty$ are distributed by hypothesis or Bayes alternative.

We prove only (3.13) in the case of Bayes alternative. In other cases the reasoning are similar.

We have

$$
\begin{aligned}
& i^{2 r} \sum_{j=i}^{\infty}\left(\eta_{j}(\delta)+y_{j} \gamma_{j}(\delta) \epsilon^{-1}\right)^{2}=i^{2 r} \sum_{j=i}^{\infty} \eta_{j}^{2}(\delta) \\
& +i^{2 r} \sum_{j=i}^{\infty} \eta_{j}(\delta) y_{j} \gamma_{j}(\delta) \epsilon^{-1}+i^{2 r} \sum_{j=i}^{\infty} y_{j}^{2} \gamma_{j}^{2}(\delta) \epsilon^{-2}=J_{1 i}+J_{2 i}+J_{3 i}
\end{aligned}
$$

The probability under consideration for the first addendum has been estimated in Lemma 2.1.

We have

$$
J_{2 i} \leq J_{1 i}^{1 / 2} J_{3 i}^{1 / 2}
$$

Thus it remains to show that, for any $C$,

$$
P_{\eta(\delta)}\left(\sup _{i} i^{2 r} \sum_{j=i}^{\infty} y_{j}^{2} \gamma_{j}^{4}(\delta) \epsilon^{-2}>\delta / C\right)=o(1)
$$

as $\epsilon \rightarrow 0$.

Note that $y_{j}=\zeta_{j}+\epsilon \xi_{j}$ where $\zeta_{j}, y_{j}, 1 \leq j<\infty$ are i.i.d. Gaussian random variables, $E \zeta_{j}=0, E \zeta_{j}^{2}=\kappa_{j}^{2}(\delta), E \xi_{j}=0, E \xi_{j}^{2}=1$.

Hence, we have

$$
\begin{aligned}
& \sum_{j=i}^{\infty} y_{j}^{2} \gamma_{j}^{4}(\delta) \epsilon^{-2}=\sum_{j=i}^{\infty} \gamma_{j}^{4}(\delta) \epsilon^{-2} \zeta_{j}^{2}+\sum_{j=i}^{\infty} \gamma_{j}^{4}(\delta) \epsilon^{-1} \zeta_{j} \xi_{j} \\
& +\sum_{j=i}^{\infty} \gamma_{j}^{4}(\delta) \xi_{j}^{2}=I_{1 i}+I_{2 i}+I_{3 i} .
\end{aligned}
$$

Since $\gamma_{j}^{2} \epsilon^{-2}=o(1)$, the estimates for probability of $i^{2 r} I_{1 i}$ are evident. It suffices to follow the estimates of (3.4). We have $I_{2 i} \leq I_{1 i}^{1 / 2} I_{3 i}^{1 / 2}$. Thus it remains to show that, for any $C$

$$
P_{\eta(\delta)}\left(\sup _{i} i^{2 r} \sum_{j=i}^{\infty} \gamma_{j}^{4}(\delta) \xi_{j}^{2}>\delta / C\right)=o(1)
$$

as $\epsilon \rightarrow 0$. Since $\gamma_{j}^{2}=\kappa_{j}^{2}(1+o(1))=o(1)$, this estimate is also follows from estimates (3.4).

Proof of Lemma 2.7 By Lemmas 2.2, 2.3 and 2.5, it suffices to show that

$$
\inf _{\theta} \sum_{j=1}^{\infty} \kappa_{j}^{2} \theta_{j}^{2}=\sum_{j=1}^{\infty} \kappa_{j}^{2}
$$

Denote $u_{k}=k^{2 r} \sum_{j=k}^{\infty} \theta_{j}^{2}$. Note that $u_{k} \leq P_{0}$. 
Then $\theta_{j}^{2}=u_{j} j^{-2 r}-u_{j+1}(j+1)^{-2 r}$. Hence we have

$$
\begin{aligned}
& A(\theta)=\sum_{j=1}^{\infty} \kappa_{j}^{2} \theta_{j}^{2}=\kappa^{2} \sum_{j=1}^{k_{\epsilon}} \theta_{j}^{2}+\sum_{j=k_{\epsilon}}^{\infty} \kappa_{j}^{2}\left(u_{j} j^{-2 r}-u_{j+1}(j+1)^{-2 r}\right) \\
& =\kappa^{2} \sum_{j=1}^{k_{\epsilon}} \theta_{j}^{2}+\kappa^{2} u_{k_{\epsilon}} k_{\epsilon}^{-2 r}+\frac{P_{0}}{r} \sum_{j=k_{\epsilon}+1}^{\infty} u_{j}\left(j^{-4 r-1}-(j-1)^{-2 r-1} j^{-2 r}\right) .
\end{aligned}
$$

Since $j^{-4 r-1}-(j-1)^{-2 r-1} j^{-2 r}$ is negative, then $\inf A(\theta)$ is attained for $u_{j}=P_{0}$ and therefore $\theta_{j}^{2}=\kappa_{j}^{2}$ for $j>k_{\epsilon}$.

Thus the problem is reduced to the solution of the following problem

$$
\inf _{\theta_{j}} \kappa^{2} \sum_{j=1}^{k_{\epsilon}} \theta_{j}^{2}+\sum_{j=k_{\epsilon}+1}^{\infty} \kappa_{j}^{4}
$$

if

and

$$
\sum_{j=1}^{k_{\epsilon}} \theta_{j}^{2}+\sum_{j=k_{\epsilon}+1}^{\infty} \kappa_{j}^{2}=\rho_{\epsilon}
$$

$$
k^{2 r} \sum_{j=k}^{\infty} \theta_{j}^{2}<P_{0}, \quad 1 \leq j<\infty,
$$

with $\theta_{j}^{2}=\kappa_{j}^{2}$ for $j \geq k_{\epsilon}$.

It is easy to see that this infimum is attained if $\kappa_{j}^{2}=\theta_{j}^{2}=\kappa^{2}$ for $j \leq k_{\epsilon}$.

\section{REFERENCES}

[1] Comminges, L. and Dalalyan, A.S. (2013). Minimax testing of a composite null hypothesis defined via a quadratic functional in the model of regression. Electronic Journal of Statistics 7 146-190.

[2] Ermakov, M.S. Minimax detection of a signal in a Gaussian white noise. Theory Probab. Appl., 35 (1990), 667-679.

[3] Hsu D., Kakade S.M., Zang T. 2012 A tail inequality for quadratic forms of subgaussian random vector. Electronic Commun. Probab. 17 No 52 p.1 - 6.

[4] Ingster, Yu.I., Suslina I.A. (2002). Nonparametric Goodness-of-fit Testing under Gaussian Models. Lecture Notes in Statistics 169 Springer N.Y.

[5] Johnstone I M 2015 Gaussian estimation. Sequence and wavelet models. Book Draft http://statweb.stanford.edu/ imj/

[6] Pinsker M S 1980 Optimal filtering of square integrable signals in Gaussian white noise. Problems of Information Transmission, 16, 120-133.

[7] Tsybakov A 2009 Introduction to Nonparametric Estimation. Springer Series in Statistics 130 Springer Berlin.

[8] Rivoirard V 2004 Maxisets for linear procedures. Statist. Probab. Lett. 67 267-275 